\title{
Can Nuclear Medicine Technologists Assess Whether a Myocardial Perfusion Rest Study Is Required?
}

\author{
Lena Johansson $^{1,2}$, Milan Lomsky ${ }^{1}$, Peter Gjertsson ${ }^{1}$, Maria Sallerup-Reid ${ }^{1}$, Johanna Johansson ${ }^{1}$, \\ Nils-Göran Ahlin ${ }^{2}$, and Lars Edenbrandt ${ }^{1}$ \\ ${ }^{1}$ Department of Clinical Physiology, Sahlgrenska University Hospital, Göteborg, Sweden; and ${ }^{2}$ Department of Clinical Physiology, \\ Central Hospital, Karlstad, Sweden
}

Both stress and rest imaging are usually performed to diagnose ischemia or infarction in the left ventricle. If the stress study is performed first and the images indicate normal findings, it might be unnecessary to perform the rest study. The current study determines whether nuclear medicine technologists can assess the necessity of a rest study. Methods: The results of gated SPECT performed using a 2-d nongated stress and gated rest $99 \mathrm{~m} T c$-sestamibi protocol for 532 consecutive patients were studied. Myocardial perfusion imaging was performed for diagnosing coronary artery disease (CAD) in 421 patients and for managing known CAD in 107 patients; 4 patients were examined for other reasons. Seventy-nine patients had previous myocardial infarction. Visual interpretation by 1 experienced physician at the time of clinical reporting was used as the gold standard for determining the scintigraphic presence of myocardial infarction or ischemia; rest, stress, and gated rest images and clinical information were available to this physician. All cases categorized as infarction or ischemia present or probably present were categorized as the group requiring a rest study (i.e., the "rest-study-required group"), whereas all other cases were categorized as the group not requiring a rest study (i.e., the "no-reststudy-required group"). A total of 3 physicians and 3 technologists independently interpreted the nongated stress images (slice images and polar plots) and decided whether a rest study was required. Results: In the rest-study-required group, the 3 technologists correctly classified on average 171 of the 172 cases, and the 3 physicians correctly classified 169 (a difference that was not statistically significant). In the no-rest-study-required group, the physicians correctly classified $32 \%$ and the technologists $21 \%$ of the cases $(P=0.001)$. The risk that a patient sent home without a rest study would have been diagnosed with infarction or ischemia using the combined stress-rest interpretation was $1.3 \%(1 / 75)$ for the technologists and $2.6 \%(3 / 115)$ for the physicians. Conclusion: The nuclear medicine technologists were able to assess whether a rest study was needed; the risk that this assessment would be incorrect was not higher for the technologists than it was for the physicians. This type of assess-

Received Mar. 4, 2008; revision accepted Aug. 14, 2008.

For correspondence or reprints contact: Lena Johansson, Department of Clinical Physiology, Sahlgrenska University Hospital, SE-413 45 Göteborg, Sweden.

E-mail: lena.bi.johansson@vgregion.se

COPYRIGHT @ 2008 by the Society of Nuclear Medicine, Inc. ment by a nuclear medicine technologist could be of value in efforts to improve effectiveness at a nuclear medicine clinic.

Key Words: image interpretation; radionuclide imaging; heart function tests; heart disease

J Nucl Med Technol 2008; 36:181-185

DOI: $10.2967 / j n m t .108 .052043$

\section{O} well-established routine that reduces patient radiation exposure. In a study of 148 patients, Worsley et al. demonstrated that rest images were not required if normal imaging findings had been obtained after exercise or pharmacologic stress (1). Schroeder-Tanka et al. came to the same conclusion in a larger study of 460 patients (2). In these 2 studies, $31 \%$ and $20 \%$, respectively, of the rest studies were not required. These studies also demonstrated that normal stress images occur more often in patients without previous myocardial infarction (MI) (41\% and 32\%, respectively) than in patients with previous MI (3\% and $4 \%$, respectively). In addition, in their study materials $74 \%$ and $59 \%$, respectively, of the patients had no history of MI. Gibson et al. studied stress-only imaging with attenuation correction and showed a low cardiac event rate among patients with normal stress findings (3). These results indicate that there is much to be gained by using a stress-only protocol, in terms of decreased radiation dose and imaging time for some patients and the ability of the clinic to examine more patients. The nuclear medicine technologist reviews the MPI images to ensure that the required information has been obtained and processed properly and is of the highest quality. For example, signs of patient motion should ideally be detected immediately after completion of an imaging session. Such image assessment for quality assurance is usually a task assigned to nuclear medicine technologists, but their responsibilities vary widely among different clinics and in different countries. In Sweden, some clinics have introduced a system in which nuclear medicine technologists can get a personal delegation from the responsible physician 
to report, for example, normal bone scan findings. The delegation is based on a judgment that the nuclear medicine technologist has received the proper education and has the required skills. Although the assessment of whether a rest study is needed and the patient must return for it is usually made by a physician, if this assessment could be delegated to the nuclear medicine technologist who acquires and reviews the stress images for quality assurance, clinic workflow could improve. The purpose of this study was to evaluate whether nuclear medicine technologists can assess the necessity of a rest study.

\section{MATERIALS AND METHODS}

\section{Patients}

The study group consisted of patients who underwent MPI between September 15, 2004, and September 14, 2005, at Sahlgrenska University Hospital in Gothenburg, Sweden. Patients with incomplete data and studies with technical problems were excluded. The study population comprised 532 patients, with a mean age of $62 \pm 11 \mathrm{y} ; 49 \%$ were men. Only 1 examination per patient was included. MPI was performed for diagnosing coronary artery disease (CAD) in 421 patients, for managing known CAD in 107 , and for other reasons in 4 . Seventy-nine patients $(15 \%)$ with previous MI and 98 patients (18\%) with a history of revascularization were included. Diabetes was present in $16 \%$, hypertension in $50 \%$, and hyperlipidemia in $44 \%$ of the patients; $16 \%$ of the patients were current smokers, and $30 \%$ had a family history of cardiac disease. The study was approved by the Research Ethics Committee at Gothenburg University.

\section{Radionuclide Imaging}

Patients were stressed using either maximal exercise, symptomlimited ergometry testing (53\%), or pharmacologic testing with adenosine. The exercise or pharmacologic stress was continued for at least $2 \mathrm{~min}$ after tracer injection. The gated SPECT studies were performed using a 2-d nongated stress and gated rest ${ }^{99 \mathrm{~m}} \mathrm{Tc}-$ sestamibi protocol. Stress and rest acquisition began approximately $60 \mathrm{~min}$ after injection of ${ }^{99 \mathrm{~m} T c-s e s t a m i b i}(600 \mathrm{MBq})$. Images were acquired using 2 different dual-head SPECT cameras (Infinia or Millennium VG; GE Healthcare) equipped with a low-energy, highresolution collimator. Images were acquired with the patient in a supine position, using step-and-shoot mode, a circular acquisition, a $64 \times 64$ matrix, a zoom factor of 1.28 , a pixel size of $6.9 \mathrm{~mm}, 60$ projections over $180^{\circ}, 40 \mathrm{~s}$ per projection, and an energy window of $20 \%$. In patients weighing over $90 \mathrm{~kg}$, the acquisition time per projection was increased to $55 \mathrm{~s}$. During rest acquisition, the patient was monitored using a 3-lead electrocardiogram. The acceptance window was opened to $\pm 20 \%$ of the predefined R-R interval, except for a limited number of studies in which a wider acceptance window was used. Other beats were rejected. Each R-R interval was divided into 8 equal time intervals. Gated SPECT acquisition was performed at the same time as ungated routine SPECT acquisition. An automatic motion-correction program was used in studies indicating patient motion during acquisition.

Tomographic reconstruction of nongated data was performed using filtered backprojection with a Butterworth filter having a critical frequency of $0.52 \mathrm{cycles} / \mathrm{cm}$ and a power factor of 5 . The gated data were reconstructed using filtered backprojection with a Butterworth filter having a critical frequency of 0.40 cycles $/ \mathrm{cm}$ and a power factor of order 10. No attenuation or scatter correction was used.

\section{Stress-Rest Interpretation}

Visual interpretation of the complete stress and rest images by 1 experienced physician at the time of clinical reporting was used as the gold standard. All cases categorized as infarction or ischemia present or probably present were categorized as the group requiring a rest study (i.e., the "rest-study-required group"), whereas all other cases were categorized as the group not requiring a rest study (i.e., the "no-rest-study-required group"). Rest, stress, gated images, clinical information, and electrocardiogram findings were available for this interpretation. The CEqual and QGS software packages were used to aid this visual interpretation $(4,5)$.

\section{Stress-Only Assessment}

A total of 3 nuclear medicine technologists and 3 physicians independently classified each of the 532 stress studies as studyrequired or no-rest-study-required. Two of the technologists had more than $10 \mathrm{y}$ of experience in MPI, and 1 had only $1.5 \mathrm{y}$ of experience. All 3 physicians were experienced in MPI. One of the physicians performed the gold standard interpretations 1 y before he performed the stress-only interpretations. Two technologists and 2 physicians came from one hospital and the other 2 from another hospital.

To assist the technologists in their classifications, the most experienced physician gave an MPI assessment tutorial and presented guidelines for classifying the stress-only images (Table 1). The rules comprised lower-normal count levels for men and women in different parts of the left ventricle. The criteria were not to be applied to patients with sarcoidosis, systemic sclerosis, or systemic lupus erythematosus. These groups of patients have been reported to have abnormal scan findings at rest that may normalize with pharmacologic stress $(6,7)$.

All observers reviewed the stress slice images and bull's-eye plots using the EXINI heart software (EXINI Diagnostics AB). No quantification data based on reference databases were available, but the count values in percentage of the maximal left ventricular activity were displayed on the screen when the pointer was moved to an area of interest. All observers reviewed the stress images, but only 2 of the physicians also had access to clinical information.

\section{Statistical Methods}

The $\chi^{2}$ test was used to analyze the differences in classification of patients into the rest-study-required and no-rest-study-required groups between the nuclear medicine technologists and the physicians. $P$ values of less than 0.05 were considered statistically significant.

\section{RESULTS}

The experienced physician interpreted the rest and stress images as indicating that infarction or ischemia was present or probably present (i.e., rest study required) in 172 (32\%) cases and as indicating no rest study required in the remaining 360 $(68 \%)$ of the 532 cases. The chance that a rest study was not required was much higher if the patient had no history of MI $(85 \%)$ than if the patient had a history of MI $(28 \%)$.

The observers correctly classified $99 \%$ of the patients in the rest-study-required group (Table 2). The technologists correctly classified more patients in this group (100\%) than did the physicians $(98 \%)$, but this difference was not statistically significant. In the no-rest-study-required group, the physicians produced higher numbers of correct classifications 
TABLE 1

Guidelines for Classification of Stress-Only Images

\begin{tabular}{|c|c|c|}
\hline \multirow[b]{2}{*}{ Region of heart } & \multicolumn{2}{|c|}{ Finding } \\
\hline & Men & Women \\
\hline Apex & $\begin{array}{l}\text { Small and sharp perfusion defect }(<50 \%) \text { or } \\
\text { perfusion defect large enough to be } \\
\text { seen in several slices and }<60 \%\end{array}$ & $\begin{array}{l}\text { Small and sharp perfusion defect }(<50 \%) \\
\text { or perfusion defect large enough to be } \\
\text { seen in several slices and }<60 \%\end{array}$ \\
\hline Anterior wall & Perfusion defect $<75 \%$ & $\begin{array}{l}\text { Perfusion defect }<65 \% \text { in } 12 \text { o'clock } \\
\text { position or }<60 \% \text { in } 11 \text { o'clock position }\end{array}$ \\
\hline Lateral-basal wall & Perfusion defect $<65 \%$ & Perfusion defect $<65 \%$ \\
\hline Inferior wall & Perfusion defect $<60 \%$ & Perfusion defect $<60 \%$ \\
\hline \multicolumn{3}{|c|}{$\begin{array}{l}\text { Septum } \\
\text { If maximum intensity in left } \\
\text { ventricle is in... }\end{array}$} \\
\hline Septum & Always & Count levels in other areas are $<65 \%$ \\
\hline Lateral wall & Perfusion defect $<65 \%$ & Perfusion defect $<65 \%$ \\
\hline
\end{tabular}

Rest study should be performed if 1 or more of criteria in table are met. Count levels in table are expressed as percentage of maximal counts per pixel of left ventricle.

than did the technologists ( $32 \%$ and $21 \%$, respectively; $P=$ 0.001). The risk that a patient sent home without a rest study would have been diagnosed with infarction or ischemia using the combined stress-rest interpretation was $1.3 \%$ (1/75) for the technologists and 2.6\% (3/115) for the physicians (not significant).

A total of 11 false classifications (as no rest study required) were made by the observers in patients classified as having infarction or ischemia using the clinical stressrest interpretation (Table 3). These cases came from 10 different patients; that is, only 1 case was falsely classified by 2 observers (Fig. 1B). The technologists contributed only 2 of these 11 false classifications. One technologist made none of these false classifications but could identify only $12 \%$ of the no-rest-study-required cases. In contrast, 2 of the physicians were able to identify $38 \%-39 \%$ of the no-study-required cases; on the other hand, they decided that no rest study was required in 3 and 5 cases, respectively, in which infarction or ischemia was actually present. The remaining 2 experienced technologists and 1 physician exhibited similar performance, having a low level of false

TABLE 2

Number of Correct Classifications of Stress-Only Images by 3 Physicians and 3 Nuclear Medicine Technologists

\begin{tabular}{ccc}
\hline & \multicolumn{2}{c}{ Correct classification } \\
\cline { 2 - 3 } Observer & $\begin{array}{c}\text { Rest study } \\
\text { required }(n=172)\end{array}$ & $\begin{array}{c}\text { No rest study } \\
\text { required }(n=360)\end{array}$ \\
\hline Physician 1 & $171(99 \%)$ & $70(19 \%)$ \\
\hline Physician 2 & $169(98 \%)$ & $135(38 \%)$ \\
Physician 3 & $167(97 \%)$ & $139(39 \%)$ \\
All physicians (mean) & $169(98 \%)$ & $115(32 \%)$ \\
Technologist 1 & $172(100 \%)$ & $87(24 \%)$ \\
Technologist 2 & $170(98 \%)$ & $94(26 \%)$ \\
Technologist 3 & $172(100 \%)$ & $43(12 \%)$ \\
All technologists (mean) & $171(99 \%)$ & $75(21 \%)$ \\
All observers (mean) & $170(98 \%)$ & $95(26 \%)$ \\
\hline
\end{tabular}

classifications in the infarction or ischemia group and identifying 19\%-26\% of the no-rest-study-required cases.

Two of the 10 falsely classified patients are presented in Figure 1. The first case (Fig. 1A) is that of a woman with a mild anterior and apical defect classified as ischemia "probably present" in the interpretation of both stress and rest images; that is, this was a rest-study-required case. Knowledge of her large bra size influenced 1 of the observers to interpret the stress defect as an attenuation artifact, and he incorrectly concluded that a rest study was not required.

The second case (Fig. 1B) is that of a male patient whose finding was misinterpreted as study-not-required by 2 observers. Interpretation of both stress and rest images indicated anterior ischemia as present.

\section{DISCUSSION}

\section{Main Findings}

Nuclear medicine technologists can assess that a rest study is not needed. The risk that this assessment is false is no higher for a technologist $(1.3 \%)$ than for a physician $(2.6 \%)$. The 2 experienced technologists and 1 of the physicians produced few if any false classifications in the infarction or ischemia group while identifying $19 \%-26 \%$ of the no-rest-study-required cases.

Two physicians had access to clinical data when assessing the stress images. One of these physicians exhibited performance similar to that of the 2 experienced technologists, indicating that clinical data are of minor importance to this classification task. This result is in accordance with the findings of Worsley et al. (1); they, however, proposed that patients with previous MI need to have a rest study, giving a high level of perfusion abnormalities in their patient group. Most important in the stress-only approach is to correctly identify those patients who might have MI or ischemia and therefore require a rest study. Assuming that 
TABLE 3

Patients Falsely Classified as No Rest Study Required

\begin{tabular}{|c|c|c|c|c|}
\hline Patient & Sex & Stress/rest interpretation & $\begin{array}{l}\text { Previous } \\
\text { MI }\end{array}$ & Observer \\
\hline 1 & M & Anterior ischemia & Yes & $P$ \\
\hline 2 & $\mathrm{~F}$ & $\begin{array}{l}\text { Probably anterior and } \\
\text { apical ischemia }\end{array}$ & No & $\mathrm{T}$ \\
\hline 3 & M & Anterior ischemia & Yes & $P+P$ \\
\hline 4 & M & $\begin{array}{l}\text { Anterior and apical } \\
\text { ischemia }\end{array}$ & No & $\mathrm{T}$ \\
\hline 5 & M & $\begin{array}{l}\text { Inferior, apical, and } \\
\text { lateral ischemia }\end{array}$ & No & $P$ \\
\hline 6 & M & $\begin{array}{l}\text { Apical and probably } \\
\text { lateral ischemia }\end{array}$ & No & $P$ \\
\hline 7 & $\mathrm{~F}$ & $\begin{array}{l}\text { Probably anterior and } \\
\text { apical ischemia }\end{array}$ & No & $P$ \\
\hline 8 & $M$ & Probably apical ischemia & No & $P$ \\
\hline 9 & $\mathrm{~F}$ & $\begin{array}{l}\text { Probably anterior and } \\
\text { apical ischemia }\end{array}$ & No & $P$ \\
\hline 10 & M & Probably lateral ischemia & No & $P$ \\
\hline
\end{tabular}

Note that patient 3 was misclassified by 2 observers. $\mathrm{P}=$ physician; $\mathrm{T}=$ technologist.

these patients can be identified accurately, it is of interest to minimize the number of superfluous rest studies. The physicians were significantly better than the technologists at identifying such patients (32\% vs. $21 \%$, respectively) but at the cost of a higher number of false classifications in the rest-study-required group. Both physicians and technologists achieved lower values than those $(52 \%)$ reported by Worsley et al. (1). One factor that may at least partly explain this difference is the higher proportion of women in the patient group of the present study (51\% here vs. $31 \%$ in the study by Worsley et al. (1)). Perfusion defects in the anterior wall of the left ventricle can be explained by an attenuation artifact, but this is difficult to assess from the stress images only (Fig. 1A).

\section{Clinical Implications}

If the nuclear medicine technologist who acquires and reviews the stress images could also make the stress-only assessment, clinic workflow would improve. The results of this study indicate that this is feasible. If this is to be the aim, the technologists must receive relevant education. It was, therefore, of interest to see that our approach of giving the technologists an interpretation tutorial and guidelines for classifying stress-only images (Table 1) resulted in such good performance, even for the technologist with only $1.5 \mathrm{y}$ of MPI experience. The only 2 false classifications in the rest-study-required group made by the technologists were cases meeting the criteria presented in Table 1.

\section{Study Limitation}

The gold standard for determining the scintigraphic presence of myocardial infarction or ischemia was the rest-stress interpretation made by 1 experienced physician. Ideally, the gold-standard method should be used independently of the myocardial perfusion images; this is difficult to accomplish, however, especially as it is important to have a large number of subjects who represent cases found in clinical routine. We used consecutive patients from a 12-mo period; that is, we focused on increasing the number of subjects instead of including fewer subjects using, for example, coronary angiography or cardiac events as the gold standard. Therefore, this study was not designed to assess the value of a stress-only or stress-rest MPI study in relation to a cardiac event rate or angiographic results.

The guidelines presented in Table 1 have not been validated in clinical settings with other patient populations, acquisition protocols, $\gamma$-cameras, and the like. They should, therefore, be regarded only as examples of criteria that could be used to evaluate a stress-only study. Other semiquantitative methods, such as the summed stress score, could serve as alternative approaches for this type of analysis.
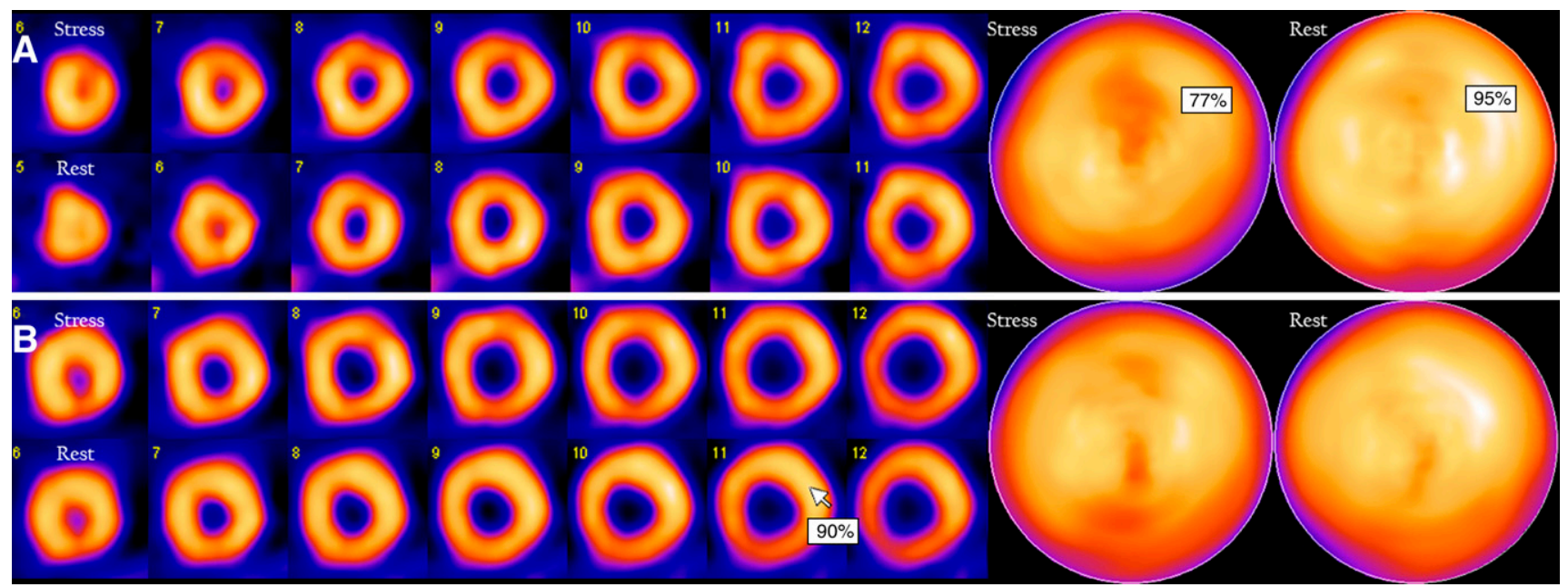

FIGURE 1. Myocardial perfusion scintigrams for 2 of 10 falsely classified patients (one shown in A and the other in B) in studyrequired group. Stress short-axis slices are above corresponding rest slices. White box in myocardium shows percentage of maximal counts per pixel of left ventricle. 


\section{CONCLUSION}

The nuclear medicine technologists were able to assess whether a rest study was needed; the risk that this assessment would be false was not any higher for the technologists than it was for the physicians. This type of assessment by a nuclear medicine technologist could be of value in efforts to improve effectiveness at a nuclear medicine clinic.

\section{REFERENCES}

1. Worsley DF, Fung AY, Coupland DB, Rexworthy CG, Sexsmith GP, Lentle BC. Comparison of stress-only vs. stress/rest with technetium-99m methoxyisobutylisonitrile myocardial perfusion imaging. Eur J Nucl Med. 1992;19: 441-444.
2. Schroeder-Tanka JM, Tiel van Buul MMC, van der Wall EE, Roolker W, Lie KI, van Royen EA. Should imaging at stress always be followed by imaging at rest in Tc-99m MIBI SPECT? Int J Card Imaging. 1997;13:323-329.

3. Gibson PB, Demus D, Noto R, Hudson W, Johnson LL. Low event rate for stressonly perfusion imaging in patients evaluated for chest pain. J Am Coll Cardiol. 2002;39:999-1004.

4. Germano G, Kiat H, Kavanagh PB, et al. Automatic quantification of ejection fraction from gated myocardial perfusion SPECT. J Nucl Med. 1995;36: 2138-2147.

5. Van Train KF, Garcia EV, Maddhai J, et al. Multicenter trial validation for quantitative analysis of same-day rest-stress technetium-99m-sestamibi myocardial tomograms. J Nucl Med. 1994;35:609-618.

6. Guludec D, Menad F, Faraggi M, Weinmann P, Battesti J-P, Valeyre D. Myocardial sarcoidosis: clinical value of technetium- $99 \mathrm{~m}$ sestamibi tomoscintigraphy. Chest. 1994;106:1675-1682.

7. Lin CC, Ding HJ, Chen YW, Wang JJ, Ho ST, Kao A. Usefulness of technetium99m sestamibi myocardial perfusion SPECT in detection of cardiovascular involvement in patients with systemic lupus erythematosus or systemic sclerosis. Int J Cardiol. 2003;92:157-161. 